\title{
Persistência de Trifloxysulfuron-Sodium e Pyrithiobac-Sodium EM Diferentes Tipos de SOLO
}

\author{
Persistence of Trifloxysulfuron-Sodium and Pyrithiobac-Sodium in Different Types of Soil \\ GUERRA, N. ${ }^{2}$, OLIVEIRA Jr., R.S. ${ }^{3}$, CONSTANTIN, J. ${ }^{3}$, OLIVEIRA NETO, A.M..$^{2}$, SANTOS, G. ${ }^{2}$ e \\ JUMES, T.M.C. ${ }^{4}$
}

\begin{abstract}
RESUMO - No Brasil, a maior parte dos cultivos de algodão encontra-se em regiões de cerrado dos Estados do Mato Grosso e Bahia, em áreas que naturalmente são constituídas de solos ácidos. Objetivou-se neste trabalho estudar a influência da acidez do solo sobre a persistência dos herbicidas trifloxysulfuron-sodium e pyrithiobac-sodium, utilizando um bioindicador como técnica de detecção. Foram conduzidos simultaneamente quatro experimentos: dois com o herbicida trifloxysulfuron-sodium $\left(7,5\right.$ e $\left.15 \mathrm{~g} \mathrm{ha}^{-1}\right)$ e dois com o pyrithiobac-sodium (70 e $\left.140 \mathrm{~g} \mathrm{ha}^{-1}\right)$. Em todos os tratamentos foi utilizado delineamento experimental inteiramente casualizado, com quatro repetições, em esquema fatorial $(3 \times 8+3)$. Os fatores estudados foram três tipos de solo (com niveis de $\mathrm{pH} 4,2,4,9$ e 5,5) e oito épocas de semeadura da espécie bioindicadora $(0,30,60,90,120,150,180$ e 210 DAA), além de três testemunhas sem aplicação do herbicida. A persistência do trifloxysulfuron-sodium na dose de $7,5 \mathrm{~g}^{\text {ha }}{ }^{-1}$ não foi influenciada pelos valores de $\mathrm{pH}$. No entanto, na dose de $15 \mathrm{~g}^{\text {ha-1}}{ }^{-1}$ a maior persistência da atividade biológica foi verificada no solo com maior $\mathrm{pH}$. Quando o pyrithiobac-sodium foi aplicado no solo com menor $\mathrm{pH}$, nas doses de 70 e $140 \mathrm{~g} \mathrm{ha}^{-1}$, o tempo necessário foi maior para que ocorresse redução da persistência. Contudo, aos 210 DAA não foram observadas diferenças na persistência do pyrithiobac-sodium $\left(70 \mathrm{~g} \mathrm{ha}^{-1}\right)$ entre diversos valores de $\mathrm{pH}$. Todavia, na dose de $140 \mathrm{~g} \mathrm{ha}^{-1}$ do pyrithiobac-sodium, o solo com maior $\mathrm{pH}$ apresentou a maior persistência desse herbicida.
\end{abstract}

Palavras-chave: bioindicador, carryover, inibidores de ALS, pH do solo, residual de herbicidas.

\begin{abstract}
In Brazil, most of the areas planted with cotton are located in the cerrado regions of Mato Grosso and Bahia, in naturally acidic soil. The objective of this work was to study the influence of soil acidity on the persistence of trifloxysulfuron-sodium and pyrithiobac-sodium, using a bioindicator as detection technique. Four experiments were conducted simultaneously: two with

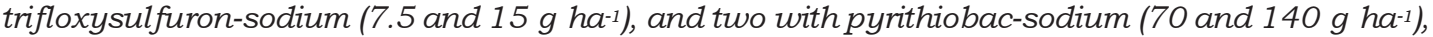
using a completely randomized design with four replications in a factorial scheme $(3 \times 8+3)$. Treatments were composed by three types of soil (at $\mathrm{pH}$ levels 4.2, 4.9 and 5.5), eight sowing dates for the bio-indicator $(0,30,60,90,120,150,180$, and $210 \mathrm{DAA})$, and three control treatments, without herbicide application. The persistence of trifloxysulfuron-sodium at doses of $7.5 \mathrm{~g} \mathrm{ha} \mathrm{a}^{-1}$ was not influenced by the $\mathrm{pH}$ values. However, at a dose of $15 \mathrm{~g} \mathrm{ha}^{-1}$ the highest increase in the persistence of biological activity was observed in soil with higher $\mathrm{pH}$. When pyrithiobac-sodium was applied to

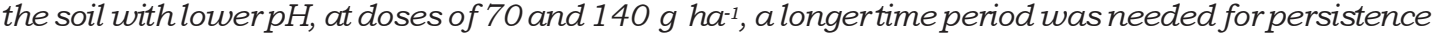
to reduce. But at 210 DAS there were no differences in the persistence of pyrithiobac-sodium $\left(70 \mathrm{~g} \mathrm{ha}{ }^{-1}\right)$ between the different $\mathrm{pH}$ values. However, at the dose of $140 \mathrm{~g}$ ha-1 of pyrithiobac-sodium, the soil with the highest $\mathrm{pH}$ showed the highest persistence of this herbicide.
\end{abstract}

Keywords: bio-indicator, carryover, ALS inhibitors, soil $\mathrm{pH}$, residual activity.

Recebido para publicação em 14.10.2010 e aprovado em 3.2.2011.

2 Discente do curso de Pós-Graduação em Agronomia na área de Proteção de Plantas, NAPD/UEM; <naiara.guerra@hotmail.com>; ${ }^{3}$ Professor Associado, Núcleo de Estudos Avançados em Ciência das Plantas Daninhas, Dep. de Agronomia, Universidade Estadual de Maringá - NAPD/UEM, Av. Colombo, 5790, 87020-9000 Maringá- PR; ${ }^{4}$ Discente do curso de Agronomia, UEM.

Planta Daninha, Viçosa-MG, v. 29, n. 3, p. 673-681, 2011 


\section{INTRODUÇÃO}

O algodão é uma planta de crescimento lento, com pequena disponibilidade de herbicidas seletivos. As áreas cultivadas com algodão são menores que as de soja e milho, envolvendo menores volumes de comercialização. O menor retorno financeiro faz com que não exista interesse por parte da indústria, pois herbicidas têm alto custo de sintese e desenvolvimento.

Trifloxysulfuron-sodium e pyrithiobacsodium são as poucas opções de herbicidas seletivos para aplicação em pós-emergência em área total, visando ao controle de plantas daninhas dicotiledôneas na cultura do algodão convencional. Esses herbicidas apresentam como mecanismo de ação a inibição da enzima acetolactato sintase (ALS) (Rodrigues \& Almeida, 2005).

O trifloxysulfuron-sodium é um herbicida do grupo das sulfonilureias, que apresenta solubilidade em água de $63 \mathrm{mg} \mathrm{L}^{-1} \mathrm{em}$ pH 5,0 e de $5.016 \mathrm{mg} \mathrm{L}^{-1} \mathrm{em} \mathrm{pH}$ 7,0. Possui um grupo funcional ionizável com um pKa de 4,76. É considerado um ácido fraco, predominando na forma neutra quando o $\mathrm{pH}$ do meio encontrase abaixo do seu pKa e na forma anionica quando o pH do meio é mais alto do que o pKa (Matocha \& Senseman, 2007). O pyrithiobacsodium pertence ao grupo químico dos ácidos pirimidiniloxibenzoicos e também é um ácido fraco, com pKa de 2,34, sendo encontrado predominantemente em sua forma anionica na maioria dos solos. É fracamente adsorvido pelos coloides do solo, apresentando coeficiente de partição $(K d)$ entre 0,22 e $0,59 \mathrm{~L} \mathrm{~kg}^{-1}$, o que sugere que essa molécula pode mover-se facilmente no perfil do solo (Baskaran \& Kennedy, 1999). A meia-vida do pyrithiobacsodium em campo é de 62 dias, e sua atividade residual no solo pode causar injúrias em culturas em sucessão, como soja, trigo e sorgo (Webster \& Shaw, 1995). Problemas de carryover relacionados ao pyrithiobac-sodium têm sido observados em solos com elevados teores de argila e baixos valores de $\mathrm{pH}$ (Smith et al., 2005).

Os herbicidas do grupo das sulfonilureias são degradados no solo devido aos processos microbiológicos e químicos, sendo este último particularmente importante em solos ácidos (Cambon et al., 1992). A degradação desse grupo de herbicidas ocorre mais rapidamente em solo não estéril que em solo estéril (Boschin et al., 2003). Devido à capacidade de dissociação em função da variação do $\mathrm{pH}$, herbicidas com características de ácidos fracos também apresentam outras propriedades além da sorção, como a solubilidade e a persistência, que variam em função da acidez do meio.

As sulfonilureias têm muitos grupos funcionais suscetiveis a diferentes reações de hidrólise. A principal reação de degradação química desse grupo de herbicidas é a clivagem das pontes dessas moléculas, a qual depende do $\mathrm{pH}$ do meio em que elas se encontram (Sarmah et al., 2000). Segundo Sarmah $\&$ Sabadie (2002), os herbicidas do grupo das sulfonilureias são hidrolisados mais rapidamente quando estão em solução aquosa ácida e ficam em sua forma estável quando em soluções aquosas neutras ou alcalinas. Esses resultados são confirmados por Matocha $\&$ Senseman (2007), os quais verificaram que o trifloxysulfuron-sodium é hidrolisado mais rapidamente em soluções aquosas com pH 5,0, se comparado a soluções com pH 7,0 e 9,0 .

No Brasil, tem-se observado frequentemente a ocorrência de intoxicação em culturas sensiveis semeadas após a utilização de herbicidas nas culturas antecessoras (Oliveira Jr., 2001; Pinto et al., 2009). A maioria das áreas cultivadas com algodão no Brasil encontra-se em regiões de cerrado dos Estados do Mato Grosso e Bahia, em áreas que naturalmente apresentam solos ácidos. Assim, objetivou-se com este trabalho investigar se a acidez do solo pode interferir na persistência da atividade biológica dos herbicidas trifloxysulfuron-sodium e pyrithiobacsodium, utilizando um bioindicador como técnica de detecção.

\section{MATERIAL E MÉTODOS}

Os experimentos foram conduzidos simultaneamente entre novembro de 2009 e julho de 2010, em casa de vegetação.

O solo original utilizado como substrato foi classificado como Latossolo Vermelho distrófico, sendo de textura franco-argiloarenosa, constituído por $71 \%$ de areia, $27 \%$ de 
argila e $2 \%$ de silte. Com relação às características químicas, apresentou $\mathrm{pH}\left(\mathrm{H}_{2} \mathrm{O}\right)$ de 4,2; $6,64 \mathrm{cmol}_{\mathrm{c}} \mathrm{dm}^{-3} \mathrm{de}^{+}+\mathrm{Al}^{3+} ; 0,43 \mathrm{cmol}_{\mathrm{c}} \mathrm{dm}^{-3} \mathrm{de}$

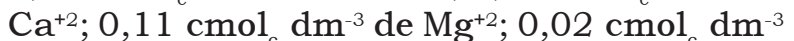
de $\mathrm{K}^{+} ; 1,2 \mathrm{mg} \mathrm{dm}^{-3}$ de $\mathrm{P}$; e $6,97 \mathrm{~g} \mathrm{dm}^{-3}$ de $\mathrm{C}$. Amostras desse solo foram coletadas na profundidade de $0-20 \mathrm{~cm}$, sendo posteriormente secas ao ar, peneiradas $(2 \mathrm{~mm})$ e acondicionadas em sacos plásticos. A acidez do solo original foi neutralizada mediante aplicações de calcário dolomítico (PRNT de 87\%) correspondentes a 2.250 e $4.500 \mathrm{~kg} \mathrm{ha}^{-1}(50$ e $100 \%$ da dose recomendada, respectivamente, para a cultura do algodão, com base na análise de solo e nas recomendações de Raij et al. (1996). Após a homogeneização do calcário no solo, as amostras foram incubadas por 90 dias, com umidade suficiente para permitir a reação do solo.

O solo, após a correção com $2.250 \mathrm{~kg}$ ha ${ }^{-1}$ de calcário dolomítico, apresentou as seguintes características químicas: $\mathrm{pH}\left(\mathrm{H}_{2} \mathrm{O}\right)$ de 4,$9 ; 5,81 \mathrm{cmol}_{c} \mathrm{dm}^{-3}$ de $\mathrm{H}^{+}+\mathrm{Al}^{3+}$; $0,61 \mathrm{cmol}_{c} \mathrm{dm}^{-3}$ de $\mathrm{Ca}^{+2} ; 0,59 \mathrm{cmol}_{\mathrm{c}} \mathrm{dm}^{-3} \mathrm{de}$ $\mathrm{Mg}^{+2} ; 0,02 \mathrm{cmol}_{\mathrm{c}} \mathrm{dm}^{-3} \mathrm{de} \mathrm{K}^{+} ; 1,4 \mathrm{mg} \mathrm{dm}^{-3}$ de P; e $6,03 \mathrm{~g} \mathrm{dm}^{-3}$ de C. Já quando recebeu $4.500 \mathrm{~kg} \mathrm{ha}^{-1}$, apresentou $\mathrm{pH}\left(\mathrm{H}_{2} \mathrm{O}\right)$ de 5,5 ; $4,21 \mathrm{cmol}_{\mathrm{c}} \mathrm{dm}^{-3} \mathrm{de} \mathrm{H}^{+}+\mathrm{Al}^{3+} ; 1,57 \mathrm{cmol}_{\mathrm{c}} \mathrm{dm}^{-3} \mathrm{de}$ $\mathrm{Ca}^{+2} ; 1,03 \mathrm{cmol}_{\mathrm{c}} \mathrm{dm}^{-3}{\mathrm{de} \mathrm{Mg}^{+2}}_{0} ; 0,03 \mathrm{cmol}_{\mathrm{c}} \mathrm{dm}^{-3}$ de $\mathrm{K}^{+} ; 1,4 \mathrm{mg} \mathrm{dm}^{-3}$ de P; e 7,31 $\mathrm{g} \mathrm{dm}^{-3} \mathrm{deC}$.

Foram conduzidos simultaneamente quatro experimentos: dois com o herbicida trifloxysulfuron-sodium (o primeiro aplicandose o herbicida na dose de $7,5 \mathrm{~g}^{-1} \mathrm{e}^{-1}$ o segundo com $15 \mathrm{~g} \mathrm{ha}^{-1}$ ) e dois com o pyrithiobacsodium (o primeiro com a dose de $70 \mathrm{~g} \mathrm{ha}^{-1}$ e o segundo com $140 \mathrm{~g} \mathrm{ha}^{-1}$ ). As doses de 7,5 $\mathrm{g} \mathrm{ha}^{-1}$ de trifloxysulfuron-sodium e 70,0 $\mathrm{g} \mathrm{ha}^{-1}$ de pyrithiobac-sodium referem-se às recomendadas para a cultura do algodoeiro (Rodrigues $\&$ Almeida, 2005). Em todos os experimentos foi utilizado delineamento experimental inteiramente casualizado, com quatro repetições, em esquema fatorial $(3 \times 8+3)$. Os fatores estudados em cada experimento foram compostos

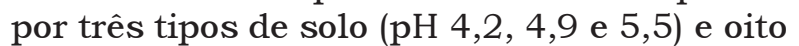
épocas de semeadura da espécie bioindicadora $(0,30,60,90,120,150,180$ e 210 dias após a aplicação dos herbicidas - DAA), além de três testemunhas de cada solo, sem aplicação do herbicida. A espécie bioindicadora utilizada foi o pepino (Cucumis sativus) (Guerra et al., 2011).
A aplicação dos herbicidas foi realizada em 02/11/2009, com um pulverizador costal pressurizado a $\mathrm{CO}_{2}$, munido de quatro pontas XR110.02, mantido à pressão de trabalho de

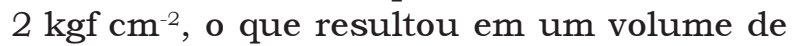
calda de $200 \mathrm{~L} \mathrm{ha}^{-1}$. No momento da aplicação, as condições ambientais eram: temperatura de $27^{\circ} \mathrm{C}$, umidade relativa do ar de $75 \%$, vento de $1,5 \mathrm{~km} \mathrm{~h}^{-1}$ e solo úmido.

Durante o tempo compreendido entre a aplicação dos herbicidas e a semeadura do bioindicador, os vasos que aguardavam a semeadura receberam duas irrigações semanais. Após cada período predeterminado, foram semeadas cinco sementes do bioindicador por unidade experimental (vasos de polietileno com capacidade de $0,75 \mathrm{dm}^{3}$ de solo), na profundidade de $1,5 \mathrm{~cm}$.

Decorridos 21 dias da semeadura do bioindicador (DAS), foram avaliadas a porcentagem de intoxicação (por meio de método visual, em que $0 \%$ corresponde a nenhum sintoma de intoxicação e $100 \%$ à morte das plantas) e a altura das plantas. O número de plantas vivas foi anotado e, em seguida, foi feito o corte da parte aérea. Esse material foi pesado em balança de precisão, a fim de se obter a matéria fresca da parte aérea. Com os dados de altura e matéria fresca da parte aérea em mãos, foram calculadas as porcentagens de inibição em relação à respectiva testemunha sem herbicida em cada solo.

Os resultados foram submetidos à análise de variância pelo teste $\mathrm{F}$ e à análise de regressão $(p \leq 0,05)$. Os modelos foram escolhidos considerando-se o coeficiente de determinação e o significado biológico. Para o herbicida trifloxysulfuron-sodium utilizou-se o modelo exponencial, e para o pyrithiobac-sodium, os modelos linear e Broken-Stick (Colwell et al., 1988).

\section{RESULTADOS E DISCUSSÃO}

Os principais sintomas de intoxicação observados nas plantas de pepino após a semeadura em solo tratado com trifloxysulfuronsodium caracterizaram-se por clorose no limbo foliar seguida de necrose nos bordos foliares, havendo, também, supressão do crescimento do bioindicador. A dose utilizada influenciou diretamente a persistência da atividade 
biológica do trifloxysulfuron-sodium, em todos os solos. Na dose de $7,5 \mathrm{~g} \mathrm{ha}^{-1}$ (Figura 1A), observaram-se sintomas mais intensos de intoxicação quando a semeadura ocorreu até 30 DAA, e nenhum sintoma a partir dos 90 DAA. Todavia, na dose de $15 \mathrm{~g} \mathrm{ha}^{-1}$ (Figura 1B) os sintomas mais evidentes ocorreram até os $60(\mathrm{pH} \mathrm{4,2} \mathrm{e} \mathrm{4,9)} \mathrm{ou} 90$ DAA (pH 5,5); nenhum sintoma foi observado a partir de 150 DAA.

Na dose recomendada para utilização na cultura do algodão, 7,5 $\mathrm{g} \mathrm{ha}^{-1}$ (Figura 1A), quando o bioindicador foi semeado imediatamente após a aplicação do herbicida, foram constatadas diferenças na intoxicação das plantas, sendo as maiores injúrias observadas no solo com $\mathrm{pH} 4,2$. No entanto, um período em torno de 60 DAA foi suficiente para reduzir as porcentagens de intoxicação para menos de $3 \%$.

Na dose de $15 \mathrm{~g} \mathrm{ha}^{-1}$ (Figura 1B) o comportamento foi diferente nos três solos; as
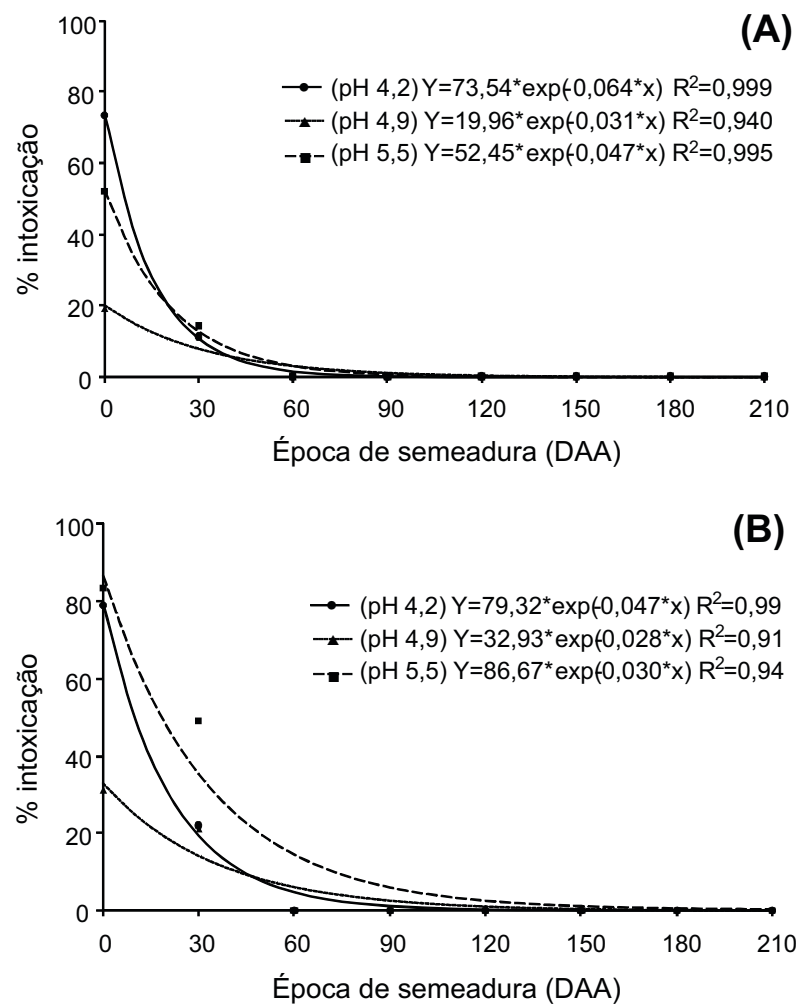

Figura 1 - Intoxicação de plantas de pepino pelo trifloxysulfuronsodium $\left[7,5 \mathrm{~g} \mathrm{ha}^{-1}\right.$ (A) e $15 \mathrm{~g} \mathrm{ha}^{-1}$ (B)], cultivadas em diferentes solos ( $\mathrm{pH} 4,2,4,9$ e 5,5) e épocas de semeadura. injúrias mais severas ocorreram no solo com maior $\mathrm{pH}$. Aos 40 DAA, os solos com $\mathrm{pH} 4,2$ e $\mathrm{pH} 4,9$ apresentaram injúrias próximas a $11 \%$, ao passo que, para que esse mesmo valor de

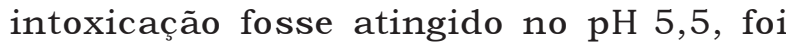
necessário um período de 70 DAA.

Na Figura 2 são apresentados os dados referentes à porcentagem de inibição da altura do bioindicador em relação à testemunha. Na dose de 7,5 $\mathrm{g} \mathrm{ha}^{-1}$ (Figura 2A), as maiores porcentagens de inibição foram observadas no solo mais ácido; quando foi aplicada a maior dose do herbicida (Figura 2B), as inibições mais intensas ocorreram no solo mais próximo da neutralidade.

Na Figura 2A, verifica-se que aos 60 DAA todos os níveis de $\mathrm{pH}$ apresentaram inibições da altura das plantas inferiores a $10 \%$, não sendo observada diferença na persistência da atividade biológica do trifloxysulfuron-sodium $\left(7,5 \mathrm{~g} \mathrm{ha}^{-1}\right)$ entre os niveis de $\mathrm{pH}$ a partir desse periodo.
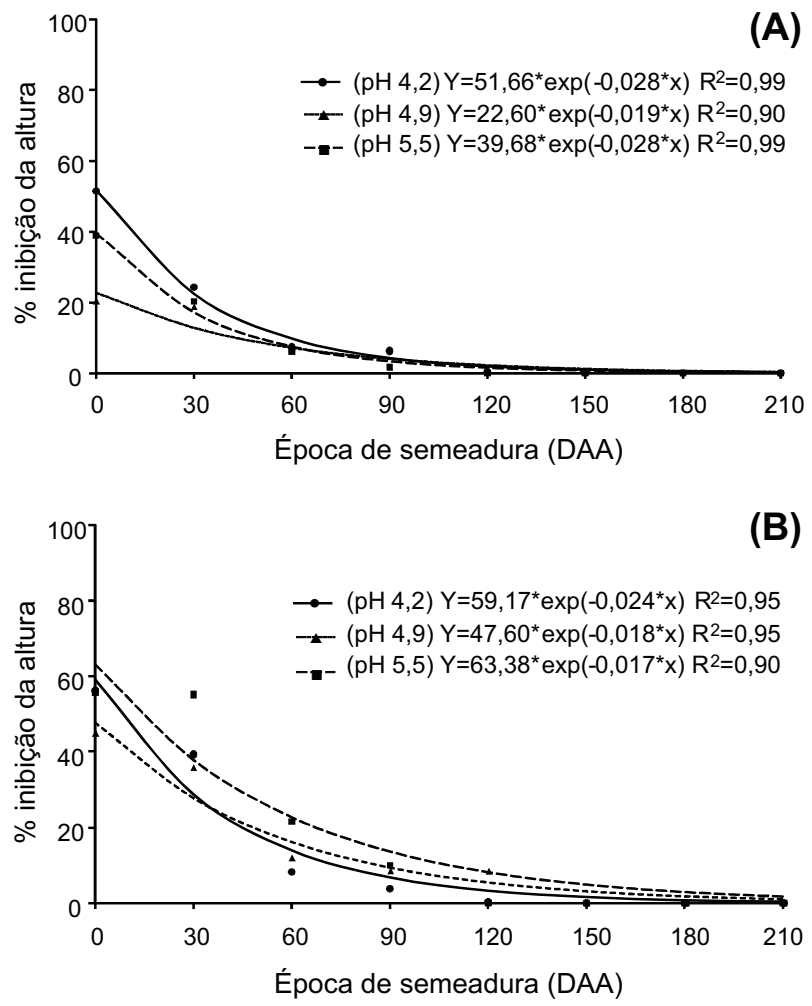

Figura 2 - Inibição da altura das plantas de pepino pelo trifloxysulfuron-sodium [7,5 $\mathrm{g} \mathrm{ha}^{-1}$ (A) e $15 \mathrm{~g} \mathrm{ha}^{-1}$ (B)], cultivadas em diferentes solos ( $\mathrm{pH} 4,2,4,9$ e 5,5) e épocas de semeadura. 
De acordo com a equação ajustada (Figura 2B), para que ocorressem inibições menores que $10 \%$ na altura das plantas, foram necessários 75, 90 e 110 dias nos solos com $\mathrm{pH} \mathrm{4,2,} \mathrm{4,9} \mathrm{e} \mathrm{5,5,} \mathrm{respectivamente.} \mathrm{O} \mathrm{aumento}$ do $\mathrm{pH}$ de 4,2 para 5,5 fez com que a persitência da atividade biológica desse herbicida fosse percebida pelo indicador por um periodo 35 dias maior. Isso se deve, provavelmente, à redução do número de sítios de ligações do solo carregados positivamente, acarretando menor sorção do herbicida e, consequentemente, aumento da sua concentração na solução do solo, o que possibilitou maior absorção pelas raízes das plantas e potencial de lixiviação em solos cujo $\mathrm{pH}$ se aproxima da neutralidade (Oliveira et al., 2005).

Os resultados da inibição da matéria fresca da parte aérea do bioindicador, em relação à testemunha sem herbicida, encontram-se na Figura 3. Verificou-se que a partir de 60 DAA a porcentagem de inibição da matéria fresca foi inferior a $10 \%$ em todos os solos (Figura 3A), e essa variável comportou-se de forma semelhante à da altura de plantas.

Quando foi aplicada a dose mais alta, foram necessários períodos de 75, 87 e 110 dias após a aplicação para que fossem atingidos valores de inibição inferiores a $10 \%$, respectivamente para os solos com pH 4,2, 4,9 e 5,5. Com isso, observou-se novamente a diferença de 35 dias na persistência da atividade desse herbicida nos solos com pH 4,2 e 5,5. O trifloxysulfuronsodium sofre degradação mais rápida em solos ácidos; a meia-vida desse herbicida em soluções aquosas com pH 5, 7 e 9 foi de 6,20 e 21 dias, respectivamente (Minton et al., 2008). $\mathrm{O}$ mesmo foi observado em outros herbicidas do grupo das sulfonilureias, como sulfosulfuron (Saha \& Kulshrestha, 2002), triflusulfuronmethyl (Vega et al., 2000), chlorsulfuron e metsulfuron-methyl (Walker et al., 1989) e flazasulfuron (Oliveira et al., 2005). Essas constatações apresentam coerência com os resultados obtidos no experimento em que se utilizaram $15 \mathrm{~g} \mathrm{ha}^{-1}$ de trifloxysulfuron-sodium, uma vez que se observou maior persistência da atividade biológica desse herbicida no solo com $\mathrm{pH}$ mais elevado.

Quando o $\mathrm{pH}$ do solo é maior que o pKa, um ácido fraco está predominantemente em sua forma aniônica, diminuindo as forças de atração entre a molécula herbicida e os coloides do solo. Além disso, os íons presentes no solo, principalmente $\mathrm{Ca}^{+2}$, podem formar complexos com as formas anionicas dos herbicidas presentes na solução do solo, enfraquecendo as forças de ligação com os coloides do solo, facilitando assim o processo de dessorção (Vivian et al., 2007). Inoue et al. (2002) verificaram que em solos com maiores niveis de calagem há lixiviação mais intensa do herbicida imazaquin, cuja dissociação no solo ocorre de forma semelhante à dos herbicidas estudados no presente trabalho, evidenciando que o aumento no $\mathrm{pH}$ proporciona menor sorção desse herbicida ao solo.

O trifloxysulfuron-sodium apresenta maior persistência em solos com $\mathrm{pH}$ mais próximo da neutralidade do que em solos mais ácidos. Esse fato pode implicar aumento no risco de carryover para culturas sensiveis a esse herbicida, como feijão (Procópio et al., 2004), milho e sorgo (Minton et al., 2008), pois a maioria dessas culturas se desenvolve melhor em solos com pH entre 5,5 e 6,5.

Na Figura 4 encontram-se as equações ajustadas para a porcentagem de intoxicação das plantas de pepino após a semeadura em solo tratado com pyrithiobac-sodium. O sintoma de intoxicação observado no bioindicador foi a intensa redução do porte das plantas.

Não foram observadas diferenças marcantes entre as doses de 70 e $140 \mathrm{~g} \mathrm{ha}^{-1}$ quando a semeadura do bioindicador foi feita no intervalo entre 0 e 90 DAA em nenhum dos solos, ficando a intoxicação entre 73,20-89,04\% e $74,40-93,80 \%$, na maior e na menor dose, respectivamente.

A porcentagem de intoxicação decresceu linearmente com o aumento do período de tempo após a semeadura no solo com pH 5,5, para ambas as doses de pyrithiobac-sodium. A taxa diária de decréscimo na intoxicação foi de 0,18 e $0,22 \%$, respectivamente, nas doses de 70 e $140 \mathrm{~g} \mathrm{ha}^{-1}$.

De acordo com o parâmetro $\mathrm{P}$ do modelo de Broken-Stick, notou-se que os sintomas de intoxicação das plantas começaram a decrescer a partir de 83 e 87 DAA de $70 \mathrm{~g}^{\text {ha }}{ }^{-1}$, nos solos com pH 4,2 e 4,9, respectivamente. Quando foi utilizada a maior dose do herbicida, foram necessários 102 DAA para que se iniciasse a 

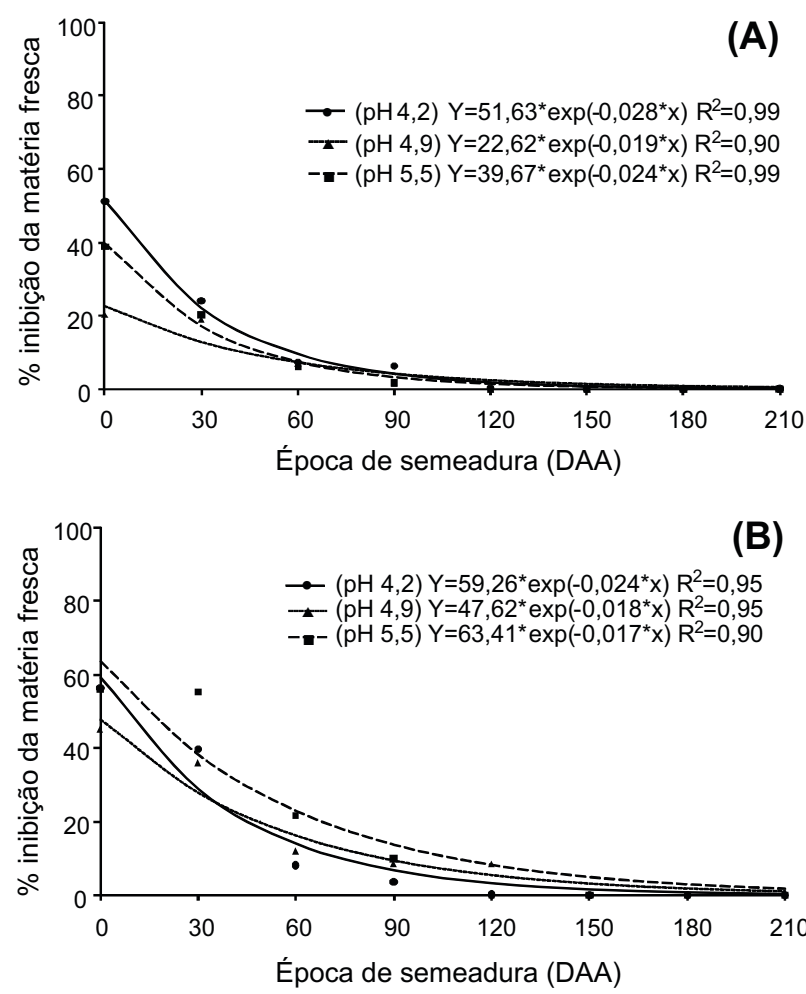

Figura 3 - Inibição da matéria fresca de plantas de pepino por trifloxysulfuron-sodium $\left[7,5 \mathrm{~g} \mathrm{ha}^{-1}\right.$ (A) e $15 \mathrm{~g} \mathrm{ha}^{-1}$ (B)], cultivadas em diferentes solos ( $\mathrm{pH} 4,2,4,9$ e 5,5) e épocas de semeadura.

redução da intoxicação no solo com $\mathrm{pH} 4,2$, enquanto no solo com $\mathrm{pH}$ 4,9 foram necessários 83 dias, semelhante ao observado na dose de $70 \mathrm{~g} \mathrm{ha}^{-1}$. Esses resultados estão de acordo com Smith et al. (2005), que verificaram que o pyrithiobac-sodium possui maior potencial de carryover quando aplicado em solos com baixos valores de $\mathrm{pH}$.

Na Figura 5 estão expostos os resultados de porcentagem de inibição da altura das plantas de pepino semeadas em diferentes épocas após a aplicação de pyrithiobac-sodium nos solos tratados com 70 (Figura 5A) e $140 \mathrm{~g} \mathrm{ha}^{-1}$ (Figura 5B). Na Figura 5A, observa-se decréscimo linear da porcentagem de inibição da altura do pepino no solo de $\mathrm{pH} 5,5$, com taxa diária de decréscimo de $0,15 \%$, quando comparado à testemunha. Nos demais solos os dados foram ajustados pelo modelo de BrokenStick. Nos solos com pH 4,2 e 4,9, foram necessários 108 e 75 DAA para que fossem observados decréscimos na inibição da altura das plantas de pepino, ocorrendo diferença de 33 dias entre esses dois solos.
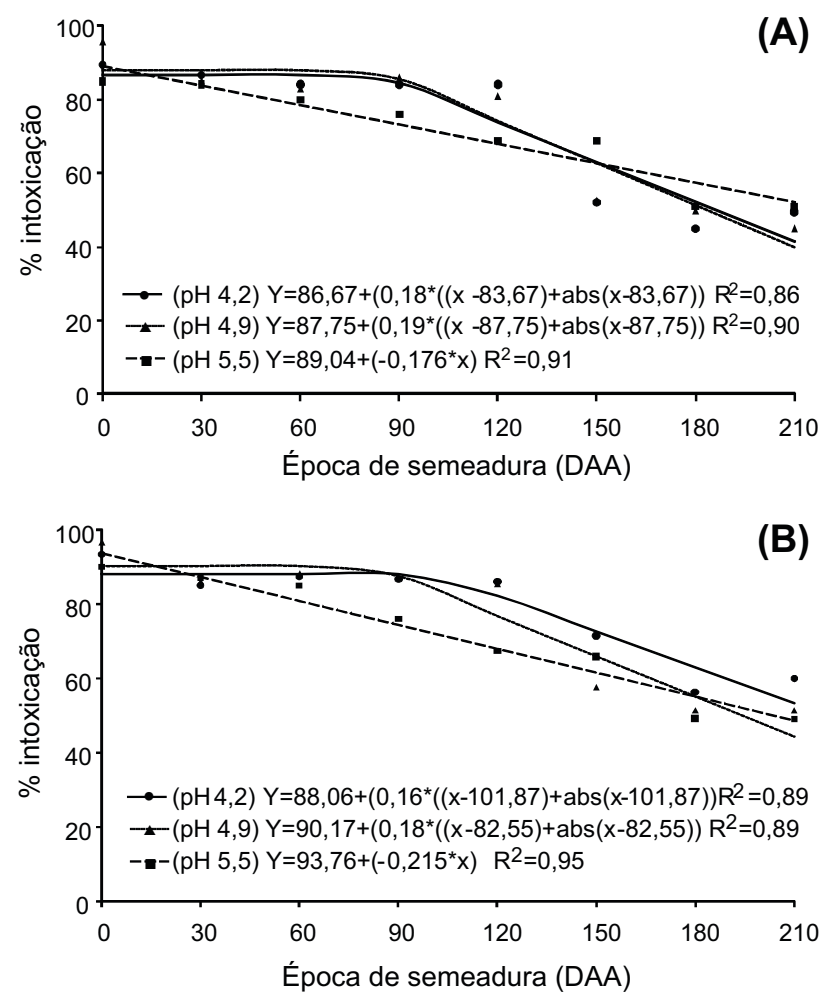

Figura 4 - Intoxicação de plantas de pepino pelo pyrithiobacsodium $\left[70 \mathrm{~g} \mathrm{ha}^{-1}\right.$ (A) e $140 \mathrm{~g} \mathrm{ha}^{-1}$ (B)], cultivadas em diferentes solos ( $\mathrm{pH} 4,2,4,9$ e 5,5) e épocas de semeadura (B).

Apesar de o solo com menor $\mathrm{pH}$ precisar de um período mais longo para iniciar a redução na inibição da altura das plantas do bioindicador, após esse período, este apresentou redução mais acentuada, chegando aos 210 DAA com porcentagens de inibição muito próximas às dos solos com $\mathrm{pH}$ de 4,9 e 5,5.

Mesmo quando semeado aos 210 DAA, o pepino ainda apresentava entre 49,3 e $53,4 \%$ de inibição após o tratamento com $70 \mathrm{~g}^{\mathrm{h}} \mathrm{a}^{-1} \mathrm{e}$ de 37,0 a 50,0\% após a aplicação de $140 \mathrm{~g} \mathrm{ha}^{-1}$ de pyrithiobac-sodium. Esses fatos indicam o potencial de utilização dessa espécie como indicador biológico da presença desse herbicida no solo, assim como a longa persistência da atividade residual deste.

Comportamento semelhante foi observado nos solos tratados com a dose de $140 \mathrm{~g} \mathrm{ha}^{-1} \mathrm{de}$ pyrithiobac-sodium. Nos solos com pH 4,2, 4,9 e 5,5 foram necessários 136, 114 e 65 DAA, respectivamente, para iniciar a redução na porcentagem de inibição. No solo com $\mathrm{pH} 4,2$, foi necessário um tempo 71 dias maior do que 
o do solo com pH 5,5 para que a persistência da atividade biológica começasse a ser efetivamente reduzida.

Contudo, após o período em que a porcentagem de inibição foi constante, no solo com $\mathrm{pH} 4,2$, o decréscimo passou a ser mais acentuado, chegando aos 210 DAA com porcentagem de inibição menor que a dos solos com pH 4,9 e 5,5.

Quanto à porcentagem de inibição da matéria fresca da parte aérea, notou-se que, sendo a semeadura realizada no mesmo dia da aplicação de pyrithiobac-sodium $\left(70 \mathrm{~g} \mathrm{ha}^{-1}\right)$ (Figura 6A), não ocorreram diferenças marcantes dessa variável nos diferentes tipos de solo. As porcentagens de inibição mantiveramse constantes até os 108,75 e 94 DAA para os solos com $\mathrm{pH} 4,2,4,9$ e 5,5, respectivamente. Após esse período, esses valores mantiveramse muito próximos, praticamente não havendo diferença entre os diversos solos aos 210 DAA.

No experimento com a maior dose de pyrithiobac-sodium (Figura 6B), o comportamento da inibição na matéria fresca da
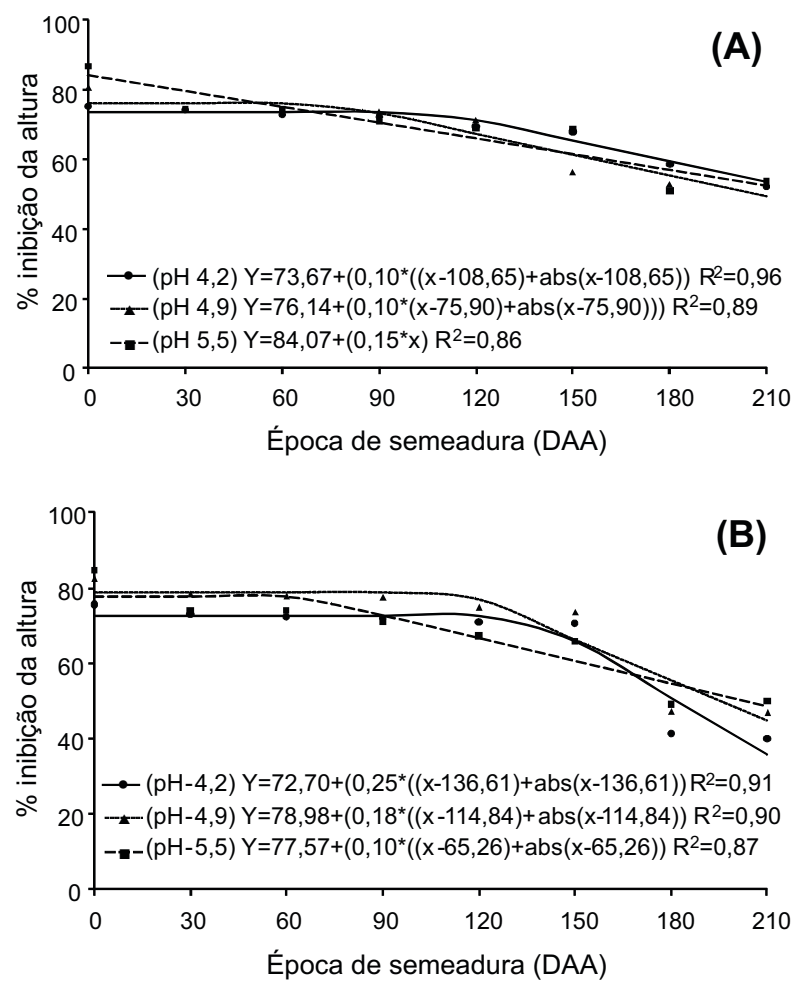

Figura 5 - Inibição da altura de plantas de pepino pelo pyrithiobac-sodium [70 $\mathrm{g} \mathrm{ha}^{-1}$ (A) e $140 \mathrm{~g} \mathrm{ha}^{1}$ (B)], cultivadas em diferentes solos ( $\mathrm{pH}$ 4,2, 4,9 e 5,5) e épocas de semeadura. parte aérea foi semelhante ao observado na altura de plantas. Quando a semeadura foi realizada imediatamente após a aplicação do herbicida, a menor porcentagem de inibição foi observada naquele com $\mathrm{pH} 4,2$ (72,68\%); nos outros solos esse valor ficou em torno de $78 \%$. Essas porcentagens de inibição mantiveram-se constantes até os 136, 131 e 67 DAA, nos solos com pH 4,2; 4,9 e 5,5, respectivamente. A persistência da atividade biológica do pyrithiobac-sodium no solo com $\mathrm{pH} 4,2$ manteve-se constante por um período de 69 dias a mais que no solo com pH 5,5.

Todavia, na última época de semeadura (210 DAA), a porcentagem de inibição foi maior no solo com pH 5,5 (49\%), seguida daqueles solos com pH 4,9 (42\%) e 4,2 (36\%). Isso mostra que o solo com $\mathrm{pH} 4,2$ demorou 69 dias mais que o com pH 5,5 para iniciar a redução da inibição da matéria fresca; após esse periodo, este apresentou redução mais intensa que os demais niveis de $\mathrm{pH}$.

Mesmo aos 210 dias após a aplicação do pyrithiobac-sodium (independentemente da
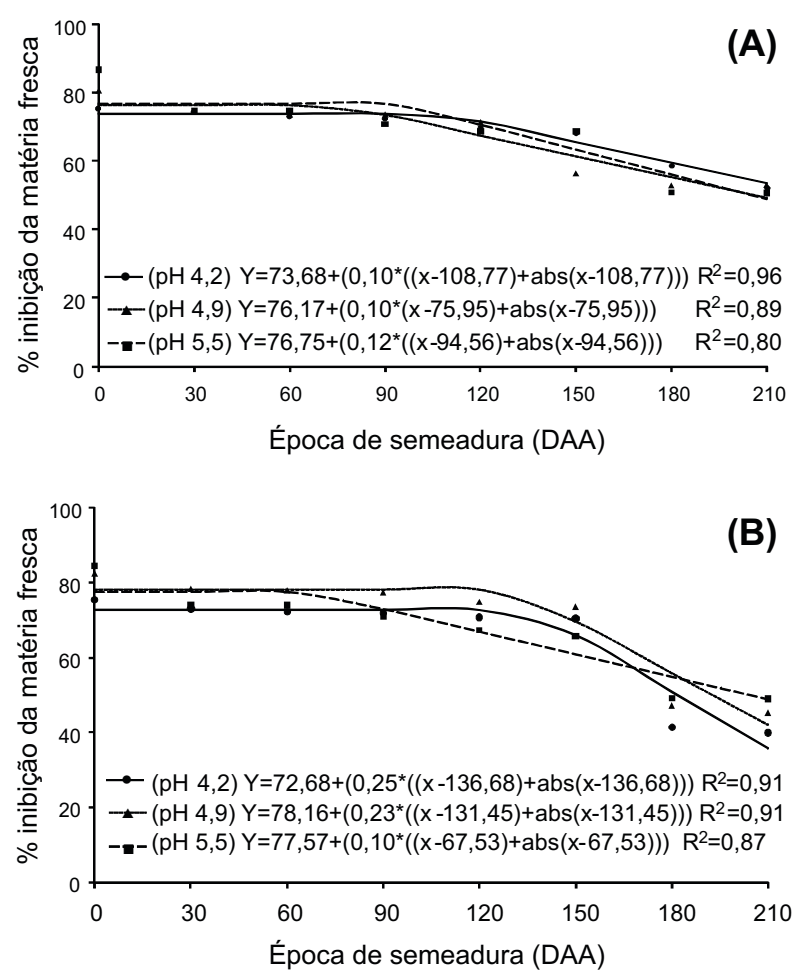

Figura 6 - Inibição da matéria fresca de plantas de pepino pelo pyrithiobac-sodium [70 $\mathrm{g} \mathrm{ha}^{-1}$ (A) e $140 \mathrm{~g} \mathrm{ha}^{-1}$ (B)], cultivadas em diferentes solos ( $\mathrm{pH} 4,2,4,9$ e 5,5) e épocas de semeadura. 
dose utilizada), ainda foi possivel detectar sua atividade no solo. No entanto, em campo, a persistência desse herbicida não é observada por períodos tão longos, o que pode ser resultado da sua mobilidade no perfil do solo (Matocha \& Hossner, 1998, 1999; Veletza et al., 2005).

Nas condições em que foram conduzidos os ensaios, pode-se concluir que a persistência da atividade biológica do trifloxysulfuronsodium avaliada com base no bioindicador foi proporcional à dose utilizada, sendo perceptivel até os 90 e 150 DAA com a aplicação de 7,5 e $15 \mathrm{~g} \mathrm{ha}^{-1}$, respectivamente. O pyrithiobacsodium promoveu intensa intoxicação até 210 DAA, independentemente da dose. A persistência do trifloxysulfuron-sodium na dose de $7,5 \mathrm{~g}^{\text {ha }}{ }^{-1}$ não foi influenciada pelo $\mathrm{pH}$ dos solos estudados. Contudo, nos solos com maior $\mathrm{pH}$, a aplicação de $15 \mathrm{~g} \mathrm{ha}^{-1}$ do trifloxysulfuron-sodium resultou em maior persistência da sua atividade biológica. Quando o pyrithiobac-sodium foi aplicado nas doses de 70 e $140 \mathrm{~g} \mathrm{ha}^{-1}$ no solo com menor $\mathrm{pH}$, foi necessário maior período para iniciar a redução na persistência. Diferentemente, aos 210 DAA não foram observadas diferenças na persistência desse herbicida na dose de 70,0 $\mathrm{g} \mathrm{ha}^{-1}$, entre os diferentes solos. A maior persistência do pyrithiobac-sodium ocorreu no solo com maior valor de $\mathrm{pH}$, tratado com 140,0 $\mathrm{g} \mathrm{ha}^{-1}$ desse herbicida.

\section{LITERATURA CITADA}

BASKARAN, S.; KENNEDY, I. R. Sorption and desorption kinetics of diuron, fluometuron, prometryn, and pyrithiobacsodium in soils. J. Environ. Sci. Health Part B Pestic. Food Contam. Agric. Wastes, v. 34, n. 6, p. 943-963, 1999.

BOSCHIN, G. et al. Biodegradation of chlorsulfuron and metsulfuron-methyl by Aspergillus niger in laboratory conditions. J. Environ. Sci. Health Part B Pestic. Food Contam. Agric. Wastes, v. 38, n. 6, p. 737-746, 2003.

CAMBON, J. P.; ZHENG, S. Q.; BASTIDE, J. Chemical or microbiological degradation of sulfonylurea herbicides in soil. I. The case of sulfometuron-methyl. Weed Res., v. 32, n. 1, p. 1-7, 1992.

COLWEELL, J. D.; SUHET, A. R.; RAIJ, B.van. Statistical procedures for developing general soil fertility models for variable regions. Meibourne: CSIRO, 1988. 68 p.
GUERRA, N. et al. Seleção de espécies bioindicadoras para os herbicidas trifloxysulfuron-sodium e pyrithiobac-sodium Rev. Bras. de Herbicidas, v.10, n.1, p.37-48, 2011

INOUE, M. H. et al. Calagem e o potencial de lixiviação de imazaquin em colunas de solo. Planta Daninha, v. 20, n. 1, p. 125-132, 2002.

MATOCHA, C. J.; HOSSNER, L. R. Pyrithiobac sorption on reference sorbents and soils. J. Agric. Food Chem., v. 46, n. 10, p. 4435-4440, 1998.

MATOCHA, C. J.; HOSSNER, L. R. Mobility of the herbicide pyrithiobac through intact soil columns. J. Agric. Food Chem., v. 47, n. 4, p. 1755-1759, 1999.

MATOCHA, M. A.; SENSEMAN, S. A. Trifloxysulfuron dissipation at selected $\mathrm{pH}$ levels and efficacy on Palmer amaranth (Amaranthus palmeri). Weed Technol., v. 21, n. 3, p. $674-677,2007$.

MINTON, B. W.; MATOCHA, M. A.; SENSEMAN, S. A. Rotational crops response to soil applied trifloxysulfuron. Weed Technol., v. 22, n. 3, p. 425-430, 2008.

OLIVEIRA JR., R. S. Atividade residual no solo de imazaquin e alachlor+atrazine visando plantio sequencial de canola. Ci. Rural, v. 31, n. 2, p. 217-222, 2001.

OLIVEIRA, M. F.; PRATES, H. T.; SANS, L. M. A. Sorção e hidrólise do herbicida flazasulfuron. Planta Daninha, v. 23, n. 1, p. 101-113, 2005.

PINTO, J. J. O. et al. Milho (Zea mays) como espécie bioindicadora da atividade residual de (imazethapyr+imazapic). Planta Daninha, v. 27, p. 1005-1014, 2009. (Número Especial)

PROCÓPIO, S. O. et al. Potencial de espécies vegetais para a remediação do herbicida trifloxysulfuron-sodium.

Planta Daninha, v. 23, n. 1, p. 9-16, 2004.

RAIJ, B.van et al. Recomendações de adubação e calagem para o Estado de São Paulo. 2.ed. Campinas: IAC, 1996. $285 \mathrm{p}$.

RODRIGUES, B. N.; ALMEIDA, F. S. Guia de herbicidas. 5.ed. Londrina: Ed. dos Autores, 2005. 591 p.

SAHA, A.; KULSHRESTHA, G. Degradation of sulfosulfuron, a sulfonylurea herbicide, as influenced by abiotics factors. J. Agric. Food Chem., v. 50, n. 16, p. $4572-4575,2002$.

SARMAH, A. K. et al. Hydrolysis of triasulfuron, metsulfuron-methyl and chlorsulfuron in alkaline soil and aqueous solution. Pest Manag. Sci., v. 56, n. 5, p. 63-471 2000 . 
SARMAH, A. K.; SABADIE, J. Hydrolysis of sulfonylurea herbicides in soils and aqueous solutions: a review. J. Agric. Food Chem., v. 50, n. 22, p. 6253-6265. 2002.

SMITH, M. C.; SHAW, D. R.; MILLER, D. K. In-field bioassay to investigate the persistence of imazaquin and pyrithiobac. Weed Sci., v. 53, n. 1, p. 121-129, 2005.

VEGA, D. et al. Triflusulfuron-methyl dissipation in water and soil. J. Agric. Food Chem., v. 48, n. 8, p. 3733-3737, 2000

VELETZA, V. G. et al. Activity, adsorption, mobility and field persistence of pyrithiobac in three soils. Weed Sci., v. 53, n. 2 , p. $212-219,2005$.
VIVIAN, R. et al. Adsorção e dessorção de trifloxysulfuronsodium e ametryn em solos brasileiros. Planta Daninha, v. 25, n. 1, p. 97-109, 2007.

WALKER, A.; COTTERILL, E. G.; WELCH, S. J. Adsorption and degradation of chlorsulfuron and metsulfuron-methyl in soils from different depths. Weed Res., v. 29, n. 4, p. 281-287, 1989.

WEBSTER, E. P.; SHAW, D. R. Carryover potential of pyrithiobac to rotational crops on a Mississippi black belt region clay soil. Weed Technol., v. 10, n. 1, p. 140-144, 1995. 